\section{Desarrollo Consulta de Enfermería Clínica en el manejo de las enfermedades reumáticas}

\section{Señor director}

La revisión de la literatura nos demuestra que enfermería especializada, con más competencias y experiencia mejora la accesibilidad al sistema sanitario [1-2], reduce las listas de espera [1], aumenta el conocimiento y el automanejo de los pacientes sobre sus enfermedades [3-6] y conlleva una reducción de costes [1-2]. Las enfermedades reumáticas representan uno de los primeros problemas crónicos de salud de la población de Cataluña [7]. Desde el año 2011 y con la implantación del modelo de Enfermería Clínica (EC) en nuestro hospital, se desarrolla la consulta de Enfermería Clínica Experta en el área de las enfermedades reumáticas como respuesta a la demanda asistencial a un problema prevalente de salud, con la finalidad de implantar el Plan Director de enfermedades reumáticas y del aparato locomotor 2010 [7] y el Plan de Salud 2011-2015 [8]. Entre las actividades principales de esta consulta se encuentran la educación sanitaria relacionada con la patología reumática y su tratamiento, reforzar el cumplimiento terapéutico y potenciar medidas no farmacológicas; realizar pruebas de screening antes de iniciar terapias biológicas; promocionar hábitos de vida saludables; asegurar la continuidad de los cuidados de estos pacientes, realizar recuentos articulares y cuestionarios de valoración de la enfermedad, funcionales y de calidad de vida; participar en estudios clínicos y proyectos de investigación e intervenir en la resolución telefónica de dudas sobre la enfermedad, la medicación y pruebas relacionadas con su enfermedad (entre otros) [9]. En Julio 2013 iniciamos la recopilación de datos para describir y analizar la actividad asistencial de la consulta de enfermería clínica en enfermedades reumáticas en nuestro hospital. La recogida de datos se realizó a través del sistema informático (SAP-asistencial). Para la extracción y
Silvia García-Díaz Catalina Padilla Llamas², Dacia Cerdà Gabaroi" Daniel Roig Vilaseca1, Delia Reina Sanz', Paula Estrada Valentina1, Vicente Torrente Segarra1, Ramon Fíguls Poch1, Hector Corominas Macias ${ }^{1}$, Jesús Esteve Ferran $^{2}$

1 Reumatología, Consorci Sanitari Integral, (Barcelona).

2 Supervisión y Dirección de enfermería, Consorci Sanitari Integral, (Barcelona).

Correspondencia:

Silvia García Díaz

" sgdphy@yahoo.es

Teléfono de contacto: 670952578 
el análisis de los datos se utilizó el programa QlikView $^{\circledR}$. Desde Enero 2012 a Julio 2013 se realizaron un total de 2963 visitas de Enfermería Clínica en el ámbito de Consultas Externas. El número de primeras visitas fue de 820 y 2.143 fue el total de visitas sucesivas. Durante este período se realizaron 66 pruebas de tuberculina y 820 cuestionarios de valoración de la enfermedad, funcionales y de calidad de vida. Además desde Mayo 2011 a Julio 2013 el número de llamadas con resolución telefónica fue de 275 . El ratio es de 1 enfermera especializada por 6 reumatólogos en una jornada laboral completa (40h/semana), con un número de 5 consultas a la semana y una consulta extra dedicada a estudios clínicos. El tiempo de una primera visita es de 40 minutos, y de 20 minutos en visitas sucesivas. Actualmente se están utilizando los criterios de derivación del reumatólogo a enfermería desarrollados por la Sociedad Española Reumatología (SER) 2013, [10]. Durante la consulta de Enfermería Clínica experta en reumatología se realizan las siguientes actividades: educación sanitaria, seguimiento analítico, evaluación periódica del paciente y resolución de dudas, ya sea en formato presencial o a distancia. Esta consulta también, nos ha permitido un abordaje más integral, complementaria al médico especialista en Reumatología en la asistencia global del paciente reumático".

\section{Referencias}

1. Hughes RA, Carr ME, Huggett A, Thawaites CE. Review of the function of a telephone helpline in the treatment of outpatients with rheumatoid arthritis. Ann Rheum Dis 2002;61(4):341-5.

2. Newhouse RP, Stanik-Hutt J, White KM, Johantgen M, Bass EB, Zangaro G, Wilson RF, Fountain L, Steinwachs DM, Heindel L, Weiner JP. Advanced practice nurse outcomes 1990-2008: a systematic review. Nurs Econ 2011;29(5):230-50.

3. Hill J, Thorpe R, Bird H. Outcomes for patients with RA: a rheumatology nurse practitioner clinic compared to standard outpatient care. Musculoskeletal Care 2003;1(1):5-20.

4. Hill J, Bird HA, Harmer R, Wright V, Lawton C. An evaluation of the effectiveness, safety and acceptability of a nurse practitioner in a rheumatology outpatient clinic. Br J Rheumatol 1994;33(3):283 -8.

5. Barry J, McQuade C, Livingstone T. Using nurse case management to promote self-efficacy in individuals with rheumatoid arthritis. Rehabil Nurs 1998;23(6):300-4.

6. Arvidsson SB, Petersson A, Nilsson I, Andersson B, Arvidsson BI , Petersson IF, Fridlund B. A nurse-led rheumatology clinic's impact on empowering patients with rheumatoid arthritis:a qualitative study. Nurs Health Sci 2006;8(3):133-9.

7. Pla director de les malalties reumàtiques I de l'aparell locomotor, (2010). Generalitat de Catalunya, Departament de Salut.

8. Pla de salut de Catalunya, (2011-2015). Generalitat de Catalunya, Departament de Salut.

9. Grupo de Enfermería de la Sociedad Valenciana de Reumatología (GESVR), 2013. Propuesta de contenidos de una consulta de enfermería en reumatología. Consejo de Enfermería de la Comunidad Valenciana (CECOVA).

10. Gobbo M, de la Torre J, Cano L, Martinez N, et al. Criterios de derivación desde reumatología a enfermería. Reu Clin 2013:9(Supp Espec. Cong); 79.

\section{Opina sobre este artículo:}

\section{f 13 in $8+\mathbf{S}$. $t$}

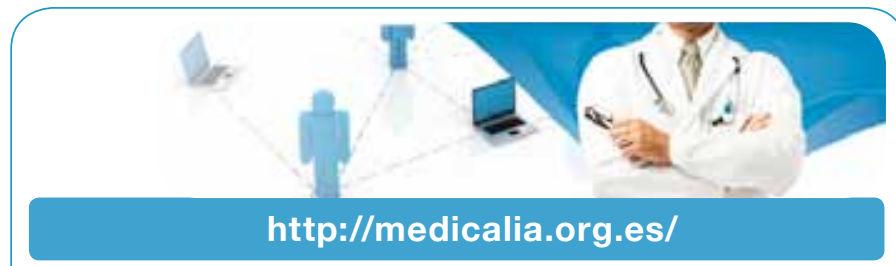

Los médicos disponen de una red social para intercambiar experiencias clínicas, comentar casos y compartir conocimiento. También proporciona acceso gratuíto a numerosas publicaciones. ¡Únase ahora!

\section{Publish with iMedPub}

http://www.imed.pub

Acta Reumatológica es una revista que tiene por fin la difusión de estudios clínicos relacionados con aspectos prácticos del diagnóstico, tratamiento y seguimiento de pacientes con patología reumatológica, de estudios epidemiológicos relacionados con patología inflamatoria y musculoesquelética de presentación común o infrecuente en la práctica clínica tanto en población adulta como pediátrica, de casos clínicos de patología poco habitual o de presentaciones inhabituales de patología frecuente, de imágenes didácticas e ilustrativas en reumatología y del estado actual e innovación en la formación especializada en reumatología. 